\title{
AVALIAÇÃO DOS MÉTODOS DE ETEST E MICRODILUIÇÃO EM CALDO PARA O ESTUDO DA SUSCETIBILIDADE DO Sporothrix schenckii COM O ITRACONAZOL
}

\author{
Ana Raquel Mano Meinerz, ${ }^{1}$ Marlete Brum Cleff, ${ }^{1}$ Patrícia da Silva Nascente, ${ }^{2}$ Luiza da Gama Osório, \\ Rafael Guerra Lund, ${ }^{3}$ Mário Carlos Araújo Meireles ${ }^{4}$ e João Roberto de Braga Mello ${ }^{1}$ \\ 1. Programa de Pós-Graduação em Ciências Veterinárias, Faculdade de Veterinária, Universidade Federal do Rio Grande do Sul (UFRGS) \\ E-mail: rmeinerz@bol.com.br \\ 2. Programa de Pós-Graduação em Veterinária, Faculdade de Veterinária, Universidade Federal de Pelotas (UFPel) \\ 3. Professor adjundo, Faculdade de Odontologia-UFPel \\ 4. Departamento de Veterinária Preventiva, Faculdade de Veterinária, UFPel.
}

\section{RESUMO}

\begin{abstract}
A frequente ocorrência de isolados fúngicos resistentes aos fármacos antifúngicos estimulou os avanços das técnicas de antifungigrama com a padronização das técnicas pelo CLSI. Porém, os métodos são ineficientes e com pouca praticidade na execução em laboratórios clínicos. Nesse contexto surgiram as técnicas comerciais, como o ETEST, que, dentre outras vantagens, demonstra maior facilidade na sua execução em relação às técnicas preconizadas pelo CLSI. O estudo utilizou o ETEST e o método de microdiluição em caldo realizado de acordo com o CLSI, para determinar a suscetibilidade de isolados de Sporothrix schenckii com o itraconazol. O CLSI emprega o meio RPMI 1640
\end{abstract}

e a leitura da CIM após o período de incubação de $72 \mathrm{~h}$ a $35^{\circ} \mathrm{C}$. No estudo, foi determinada a CIM pelo ETEST utilizando o meio ágar Sabouraud dextrose e realizando a leitura após 72 horas de incubação a $35^{\circ} \mathrm{C}$. A análise de variância feita pelo teste de T pareado não demonstrou diferenças estatísticas entre os valores das CIMs obtidos pela técnica de microdiluição em caldo (MIC entre 0,219 e $0,875 \mu \mathrm{g} / \mathrm{mL}$ ) e o ETEST (MIC entre 0,032 e $2,0 \mu \mathrm{g} / \mathrm{mL}$ ), porém o coeficiente de correlação $(\mathrm{R})$ foi negativo, provavelmente pelo pequeno número de amostras. Esses resultados estimulam mais estudos que confirmem a aplicação do ETEST para avaliar a suscetibilidade do $S$. schenckii com o itraconazol.

PALAVRAS-CHAVES: Esporotricose, microdiluição em caldo, Sporothrix schenckii, suscetibilidade.

\section{ABSTRACT}

\section{EVALUATION OF ETEST AND BROTH MICRODILUITION METHODS FOR ITRACONAZOLE SUSCEPTIBILITY TESTING OF Sporothrix schenckii}

The frequent occurrence of resistant isolated fungi against antifungal drugs stimulated advances in the antifungigram techniques, which were standardized by CLSI. However, the methods have been inefficient and impractical to be executed in clinical laboratories. Within this context, commercial techniques have been developed, being ETEST one of them. ETEST has proved to be easier to execute when compared to the techniques approved by the CLSI. This study used the ETEST and the microdilution method, performed according to CLSI, for determining the in vitro susceptibility of isolates of Sporothrix schenckii against itraconazole. The CLSI uses RPMI 1640 medium and the reading of MIC after the period of incubation of $72 \mathrm{~h}$ at $35^{\circ} \mathrm{C}$. MIC was determined by the ETEST, being Sabouraud dextrose agar used as medium, and the reading performed after 72 hours of incubation at $35^{\circ} \mathrm{C}$. The variance analysis, analyzed by T-paired test, did not demonstrate statistical differences among the CIM values obtained by the microdilution technique in broth (MIC among 0.219 and $0.875 \mu \mathrm{g} /$ $\mathrm{mL}$ ) and ETEST (MIC among 0.032 and $2.0 \mu \mathrm{g} / \mathrm{mL}$ ). However, the correlation coefficient $(\mathrm{R})$ was negative, probably because of the small number of samples. These results show the necessity of further studies to assess the application of ETEST to evaluate the susceptibility of $S$. schenckii against the itraconazol.

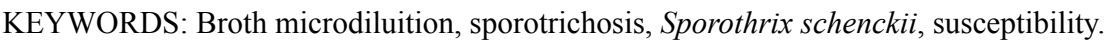




\section{INTRODUÇÃO}

A esporotricose, micose subcutânea causada pelo fungo dimórfico Sporothrix schenckii, acomete o homem e várias espécies de animais. O felino doméstico está frequentemente envolvido nos relatos zoonóticos da micose, sendo que estudos demonstram que o homem adquire a micose através de arranhadura, mordedura ou pela contaminação por solução de continuidade cutânea preexistente de animais afetados. A transmissão é facilitada pela grande quantidade de células leveduriformes presentes nas lesões cutâneas dos felinos com esporotricose (BARROS et al., 2004).

O tratamento da esporotricose em felinos é realizado, frequentemente, com itraconazol, sendo este considerado atualmente o fármaco de eleição para as formas cutâneas e linfocutâneas da micose em humanos. Porém, devido ao seu uso indiscriminado, têm sido frequentes os relatos de isolados resistentes ao fármaco, levando consequentemente a falhas terapêuticas e remissão da micose tanto nos homens como em animais (ROCHETTE et al., 2003; SCHUBACH et al., 2004).

Os crescentes relatos de isolados resistentes aos fármacos antifúngicos impulsionaram o avanço das técnicas de suscetibilidade antifúngica e, com base nos documentos descritos pelo Clinical and Laboratory Standards Institute (CLSI), foi possível padronizar a execução de testes de antifungigrama. Porém, as técnicas preconizadas pelo CLSI apresentam como desvantagens a complexidade na execução e a variabilidade inter e intralaboratoriais nos valores dos CIMs (CORMICAN \& PFALLER, 1996; ESPINELINGROF, 1996).

Nesse contexto, surgiram as provas comerciais com vantagens em relação à metodologia descrita pelo CLSI, entre elas a de oferecer resultados mais rápidos e praticidade na sua execução. Em especial o ETEST, técnica baseada na difusão do agente em ágar, consiste numa fita de papel com um gradiente de concentração do antifúngico, a qual é colocada na superfície do meio de cultivo para a obtenção da concentração inibitória mínima (CIM). Esse método já foi utilizado em várias espécies de leveduras como Candida spp. e Cryptococcus neoformans (COLOMBO et al., 1995; KOÇ et al., 2000), porém não há estudos que avaliem a suscetibilidade do $S$. schenckii através dessa técnica.
Considerando o itraconazol como um dos fármacos mais intensamente utilizados na clínica veterinária de pequenos animais, assim como a importância de detectar a suscetibilidade de isolados de $S$. schenckii com o fármaco de forma mais rápida e com maior facilidade de execução da técnica de antifungigrama, o estudo tem como objetivo avaliar o ETEST e a técnica de microdiluição em caldo na determinação da suscetibilidade do $S$. schenckii com o itraconazol.

\section{MATERIAL E MÉTODOS}

Utilizaram-se cinco isolados clínicos de $S$. schenckii provenientes de casos clínicos de esporotricose felina e humana, os quais foram mantidos na sua fase miceliana e leveduriforme através de subculturas para tubos com meio ágar Sabouraud dextrose, com cloranfenicol acrescido de cicloheximida, sendo mantidas sob refrigeração em temperatura média de $4^{\circ} \mathrm{C}$. Estudaram-se os isolados na fase leveduriforme quanto à suscetibilidade com o itraconazol (Janssen Pharmaceutical) através da técnica de microdiluição em caldo descrita no documento CLSI e pelo ETEST.

A técnica de microdiluição em caldo foi executada de acordo com o documento CLSI, em que, a partir da solução-mãe do itraconazol nas concentrações de $1.600 \mu \mathrm{g} / \mathrm{mL}$, foram realizadas nove diluições seriadas em DMSO alcançando uma concentração final dos antifúngicos de $3 \mu \mathrm{g} / \mathrm{mL}$. Diluíram-se essas concentrações a 1\% cada em meio RPMI 1640 (GIBCO), estabelecendo concentrações com intervalos de $16 \mu \mathrm{g} / \mathrm{mL}$ a 0,03 $\mu \mathrm{g} / \mathrm{mL}$, as quais foram distribuídas em microplacas no sentido das colunas em volume de $100 \mu \mathrm{L}$.

Os inóculos fúngicos utilizados para o emprego da técnica de microdiluição em caldo foram preparados a partir de colônias com até 48 horas de incubação da fase leveduriforme de $S$. schenckii, sendo ressuspendidas em tubos contendo solução salina estéril depois de homogeneizada em agitador e ajustada a turbidez em 0,5 da escala de MacFarland. Essa suspensão fúngica foi diluída em solução salina (diluição 1:50) homogeneizada em agitador e submetida à diluição em meio RPMI 1640 (diluição 1:20). Esse inóculo foi então distribuído no sentido das linhas das microplacas.

O teste de microdiluição em caldo foi realizado em duplicata, sendo uma coluna da microplaca para o controle positivo, preenchida com meio RPMI1640 e 
inoculo fúngico, e outra coluna referente ao controle negativo, contendo o meio RPMI1640. Realizou-se a interpretação dos resultados pela visualização da turvação, referente à multiplicação do microrganismo comparada a controles positivos e negativos, sendo a menor concentração capaz de produzir proeminente inibição do crescimento da levedura em relação ao poço controle positivo identificada como a concentração inibitória mínima (CIM) do fármaco para o $S$. schenckii.

Executou-se a técnica do ETEST (AB BIODISK, Salna, Sweden) de acordo com as normas do fabricante da fita. Uma amostra da colônia leveduriforme de $S$. schenckii de 48 horas de incubação foi ressuspendida em $5 \mathrm{~mL}$ de solução fisiológica estéril, com a posterior homogeneização em Vortex e ajustada na turbidez a 0,5 da escala de McFarland. Com o auxílio de um $s w a b$ estéril, o inoculo foi semeado na superfície de placas de Petri contendo ágar Sabouraud dextrose, acrescido de cloranfenicol (DifcoLaboratórios, Detroit, Mich.), com a aplicação da fita do ETEST após quinze minutos. Incubou-se a placa a $35^{\circ} \mathrm{C}$ por 72 horas, período suficiente para delimitar o halo de crescimento. A CIM foi obtida a partir do valor da escala onde a elipse de inibição intercepta a fita.

Os valores dos CIMs, obtidos a partir da execução dos testes de suscetibilidade antifúngica (ETEST e microdiluição em caldo), foram analisados estatisticamente através da análise de variância pelo teste $\mathrm{T}$ pareado e pelo cálculo do coeficiente de correlação (R).

\section{RESULTADOS E DISCUSSÃO}

Os valores da CIM para o itraconazol utilizando o ETEST variaram de 0,032 a $2.0 \mu \mathrm{g} / \mathrm{mL}$ e pela técnica de microdiluição em caldo de 0,219 a $0,875 \mu \mathrm{g} / \mathrm{mL}$ (Tabela 1). As médias de variância não demonstraram diferenças estatisticamente significativas entre os valores das CIMs conforme as técnicas de suscetibilidade empregadas $(\mathrm{p}=0,78)$, porém foi observado um coeficiente de correlação $(R)$ negativo entre os testes, provavelmente devido ao pequeno número de amostras estudadas. Outras análises que utilizaram o ETEST para avaliar a suscetibilidade de leveduras como Candida spp. e Cryptococcus neoformans com os azóis resultaram numa boa correlação entre os resultados de CIM com o método de microdiluição em caldo (ESPINEL-INGROF, 1996). Porém estudos comparando as técnicas frente a leveduras de interesse médico e várias espécies de fungos filamentosos mostraram discordância entre os valores das CIMs do itraconazol obtidos através da técnica de microdiluição em caldo e o ETEST (SZEKELEY et al., 1999; CHANG et al., 2001).

TABELA 1. Concentração inibitória mínima do itraconazol frente a isolados clínicos de Sporothrix schenckii na fase leveduriforme através do ETEST e da técnica de microdiluição em caldo (MC)

\begin{tabular}{lcc}
\hline $\begin{array}{l}\text { Sporothrix } \\
\text { schenckii }\end{array}$ & $\begin{array}{c}\text { CIM }(\mu \mathrm{g} / \mathrm{ml}) \\
\text { ETEST }\end{array}$ & $\begin{array}{c}\text { CIM }(\mu \mathrm{g} / \mathrm{ml}) \\
\text { MC }\end{array}$ \\
\hline $1 *$ & 0,032 & 0,875 \\
$2 *$ & 0,032 & 0,875 \\
$3 * *$ & 0,094 & 0,219 \\
$4 *$ & 1,0 & 0,219 \\
$5 * *$ & 2,0 & 0,219 \\
\hline
\end{tabular}

* Isolado obtido de esporotricose felina.

** Isolado obtido de esporotricose humana.

As técnicas comerciais podem ser utilizadas na determinação da suscetibilidade antifúngica se os valores da CIM dessas provas forem de acordo com os resultados obtidos a partir das técnicas recomendadas pelo CLSI. Porém, as provas de suscetibilidade empregadas para os fungos dimórficos ainda não são confiáveis, sendo que na fase filamentosa desses agentes se observa dificuldade na padronização do inóculo, enquanto que, na fase leveduriforme, o tempo de crescimento pode ser mais lento em comparação às bactérias ou às outras leveduras, dificultando a execução da técnica (ESPINEL-INGROFF, 1996).

O estudo adaptou o ETEST para a aplicação da fase leveduriforme de um fungo dimórfico, utilizando como meio de cultivo o meio ágar Sabouraud dextrose (Figura 1). NASCENTE et al. (2003) observaram que a Malassezia pachydermatis não resultava em um bom crescimento no meio RPMI 1640, optando pelo meio ágar Sabouraud dextrose para a execução dos testes de suscetibilidade do agente. Existe um desacordo sobre a eleição do meio mais apropriado para a execução dos testes de antifungigrama devido às diferenças metabólicas entre fungos filamentosos e leveduriformes, sendo 
que já foi demonstrado que as leveduras não crescem bem no meio preconizado pelo CLSI, o meio RPMI 1640, assim como determinados fungos filamentosos (REX et al., 2001).

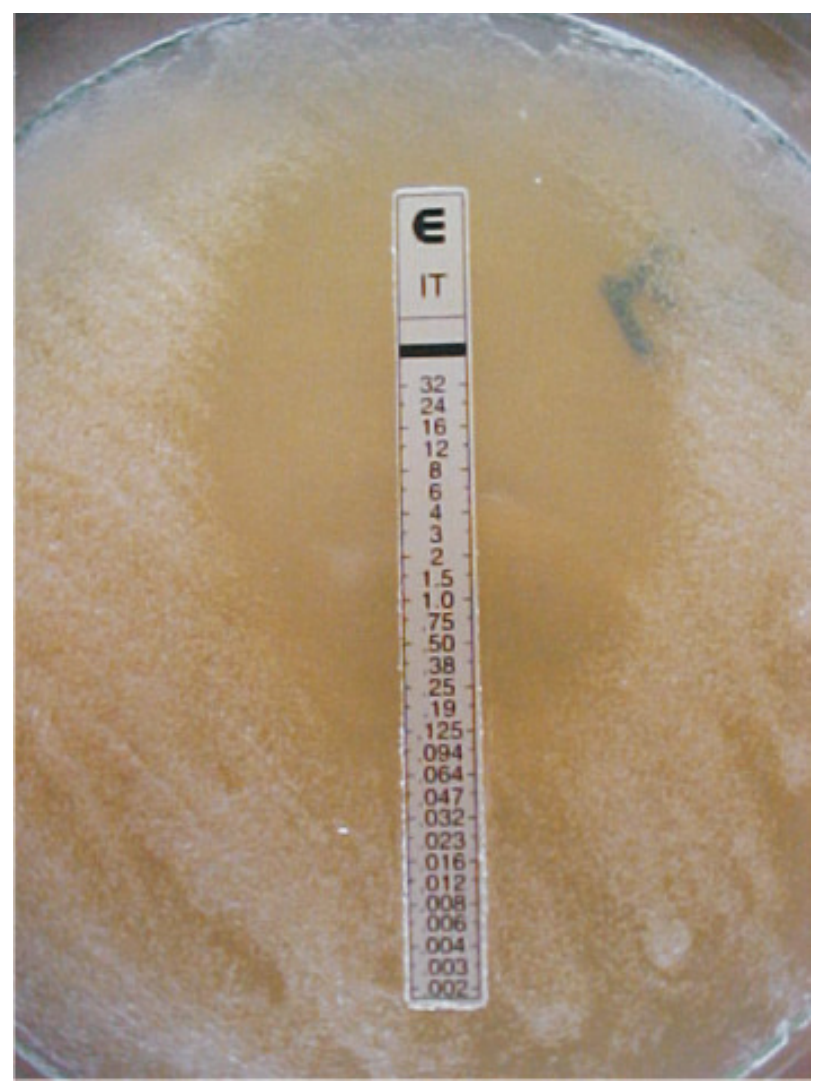

FIGURA 1. Concentração inibitória mínima (CIM) do itraconazol obtida através da técnica do ETEST frente a um isolado de Sporothrix schenckii em meio ágar Sabouraud dextrose após 72 horas a $35^{\circ} \mathrm{C}$ - Laboratório de Micologia-UFPel, 2005.

\section{CONCLUSÃO}

O estudo ressalta a necessidade de padronização de testes de suscetibilidade para fungos dimórficos, considerando a importância crescente dessa classe de microrganismos na medicina animal. Ainda considera as vantagens do uso de provas comerciais como o ETEST para determinar a sensibilidade do $S$. schenckii. Os resultados obtidos geram a necessidade de maiores estudos que consigam determinar as reais vantagens do uso do ETEST como teste rotineiro e recomendado para a avaliação da suscetibilidade do $S$. schenckii com o itraconazol.

\section{REFERÊNCIAS}

BARROS, M. B. L.; SCHUBACH, A. O.; DO VALLE, A. C. F, GALHARDO, M. C. G.; CONCEIÇÃO-SILVA, F.; SCHUBACH, T. M. P.; REIS, R. S.; WANKE, B.; MARZOCHI, K. B. F.; CONCEIÇÃO, M. J. Cat-transmitted sporotrichosis epidemic in Rio de Janeiro, Brazil: Description of a series of cases. Clinical Infectious Diseases Society of America, v. 38, p 529-535, 2004.

CHANG, H. C.; CHANG, J. J.; CHAN, S. H.; HUANG, A. H.; WU, T. L.; LIN, M. C.; CHANG, T. C. Evaluation of ETEST for direct antifungal susceptibility testing of yeasts in positive blood cultures. Journal of Clinical Microbiology, v. 39, p. 1328-1333, 2001.

COLOMBO, A.; BARCHIESI, F.; RINALDI, M. Comparision of ETEST and National Commitee for Clinical Laboratory Standars Both Macrodiluition Method for Azole Antifungal Susceptibility testing. Journal of Clinical Microbiology, v. 33, p. 335-540, 1995.

CORMICAN, M. G.; PFALLER M, A. Standardization of antifungal susceptibility testing. Journal of Antimicrobial Chemotherapy, v. 38, p. 561-578,1996.

ESPINEL-INGROF, A. Standardization of antifungal susceptibility testing: review update. Revista Iberoamerican de Micologia, v. 13, p. 64-68, 1996.

KOÇ NEDRET, A.; GOKAHM, S.; OGUZKAYA, M. Comparision of ETEST with the broth microdiluition method in susceptibility testing of yeast isolates against four antifungal. Journal of Clinical Microbiology, v. 15, p. 293-297, 2000.

NASCENTE, P.S; NOBRE, M.O.; SCHUCH, L.F.D.; LUCIA Jr.,T.; FERREIRO, L.; MEIRELES,M.C.A. Evaluation of Malassezia pachydermatis antifungal susceptibility using two different methods. Brasilian Journal Microbiology, v. 34, p. 359-362, 2003.

REX, J. H.; PFALLER, M. A.; WALSH, T. J.; CHATURVEDI, V.; ESPINEL-INGROFF, A. et al. Antifungal susceptibility testing: practical aspects and current challenges. Clinical Microbiology Reviews, v. 14, p. 643-658, 2001.

ROCHETTE, F.; ENGELEN, M.; VANDEN BOSSCHEM, H. Antifungal agents of use in animal health-pratical applications. Journal Veterinary Farmacol, v. 26, p. 31-53, 2003.

SCHUBACH, T. M. P.; SCHUBACH, A. O.; KAMOTO BARROS, T. M. B. L.; FIGUEIREDO, F. B.; CUZZI, T.; FIALHOMONTEIRO, P. C.; REIS, R. S.; PEREZ, M. A. Evaluation of an epidemic of sporotrichosis in cats: 347 cases (1998-2001). Journal of the American Veterinary Medical Association, v. 224, p. 1623-1629, 2004. 
SZEKELEY, A.; JONHSON, E. M.; WARNOCK, D. Comparision of ETEST and broth microdiluition methods for antifungal drug suscetibility testing of molds. Journal of Clinical Microbiology, v. 39, p. 1840-1843, 1999.

Protocolado em: 18 nov. 2006. Aceito em: 25 jan. 2010. 